\title{
Problem-oriented knowledge processing on the basis of hybrid approach
}

\author{
Elmar Kuliev, Yury Kravchenko, Nina Kulieva, Vladimir Kureichik \\ Southern Federal University \\ Rostov-on-Don, Russia \\ elmar_2005@mail.ru,krav-jura@yandex.ru,xolopova.nina@mail.ru,vkur@sfedu.ru
}

\begin{abstract}
The paper discusses a hybridization of adaptation mechanisms with self-organization in order to process problemoriented knowledge. The core of the proposed method consists in sequential execution of bionic and genetic algorithms. To demonstrate the hybrid algorithm we gave an example of a solution search. In the paper it is presented a formulation of the problem-oriented knowledge processing task during the optimal solution search. To solve this problem we developed a modified architecture of the hybrid search. The suggested architecture involves principal components of a bioinspired search. To improve the algorithm performance we provide an opportunity to add new algorithms with an evolutionary adaptation module and environment module. . Conducted experiments confirmed that time complexity of the developed approach is polynomial.
\end{abstract}

Keyword-genetic algorithm, evolutionary algorithm, hybrid approach, adaptation, neighborhood, population, selforganization, problem-oriented knowledge.

\section{INTRODUCTION}

At the present time amount of information increases constantly due to the so-called "curse of dimensionality". One of the main problems related to science and engineering development is the problem-oriented knowledge processing. So, it is necessary to develop theories, principles, integrated models and methods to the effective solution of problemoriented tasks. There are a lot of effective methods of knowledge processing and analysis such as simulation of evolution in nature; adaptation; hierarchical self-organization; genetic search and programming; bioinspired and genetic algorithms. [1-3]

A novel hybrid method allows to analyze, synthesize and simulate alternative solutions taking into account experience, knowledge and preferences of developers and engineers. To effective decision making the descendant population is formed on the basis of the parent population Thus, the technology of evolution simulation is transformed into a complex of alternations of generations algorithms. [2]Hence, integration of bioinspired and search methods is essential for modernization of problem-oriented knowledge. Such approach involves the use of evolution simulation methods, bioinspired and genetic algorithms, evolutionary strategies, adaptation and communication with environment [4-7].
In recent time a developing and researching of new algorithms inspired by natural systems is a priority task to the effective processing of problem-oriented knowledge. At the same there is a conflict between complexity and requirements to the effective decision making in a real time. These problems cannot be completely solved by decision making parallelization, increasing number of operators, users or decision making persons (DMP) [5-8].For this reason we suggest to use new technologies at the interface between the informatics and biological cybernetics.

There are novel tools for intelligent optimization such as ant colony optimization, particle swarm optimization, artificial bees' colony optimization, collective adaptation etc. Problem complexity is caused by the processing of big data sets. For this reason we suggest a new hybrid approach based on integration of bioinspired search methods and adaptive behavior models in terms of problem-oriented knowledge processing. Each model has metaheuristics which improve effectiveness of algorithms. This approach allows us to partially solve the problem of preliminary convergence.

\section{PROBLEM FORMULATION}

Let us formulate the task of problem-oriented knowledge processing during optimal solutions search. At the initial stage a set of objects $Z$ (a population of solutions) is denoted. Among these objects it is needed to select the best ones according with an optimality criterion F. The optimality criterion, which based on object characteristics, is represented as $\mathrm{F}: \mathrm{Z} \rightarrow \mathrm{R}$, where each objects $z \in Z$ corresponds to the value $F(x)$. The set of objects is random, and we can build coded representation of sets within a finite vector set $S$ (i.e. genotype). An expression $\varphi: \mathrm{Z} \rightarrow \mathrm{S}$ shows a connection between target objects, which are solutions of the task, and objects, which controlled by the search algorithm [4, 9-11].

During optimization the set $\mathrm{Z}$ tents to the optimal value by changing input parameters of objects. The set of objects $s \in S$ defined by the bioinspired search algorithm. The evolution of the set $\mathrm{Z}$ corresponds to the evolution of the population $\mathrm{S}$. The subset $\mathrm{P} 0$ is a random initial population defined on the $\mathrm{S}$. At each step the solution is calculated in the following way: $\mathrm{Pt}+1$ $=\mathrm{A}(\mathrm{Pt})$, where $\mathrm{A}$ is a combination of various evolutionary operators [8].The combination of evolutionary operators A 
defines the optimality criterion, which also calculated at each step.

\section{MODIFIED ARCHITECTURE OF THE HYBRID APPROACH}

In terms of problem-oriented knowledge processing a topical issue is a preliminary convergence of algorithms - in other words, falling into local optimum. Because of there is a necessary to develop new search methods and architectures, which allow to reduce computational resources and find optimal and quasi-optimal results during polynomial time.

To improve performance of problem-oriented knowledge processing it is expedient to apply evolutionary modeling, genetic search and principles of adaptation and selforganization [12].

For this purpose we developed a new modified architecture of the hybrid approach (Fig. 1). Here input parameters are required constructive restrictions, control parameters of the genetic algorithm such as size of population, probabilities of genetic operators.

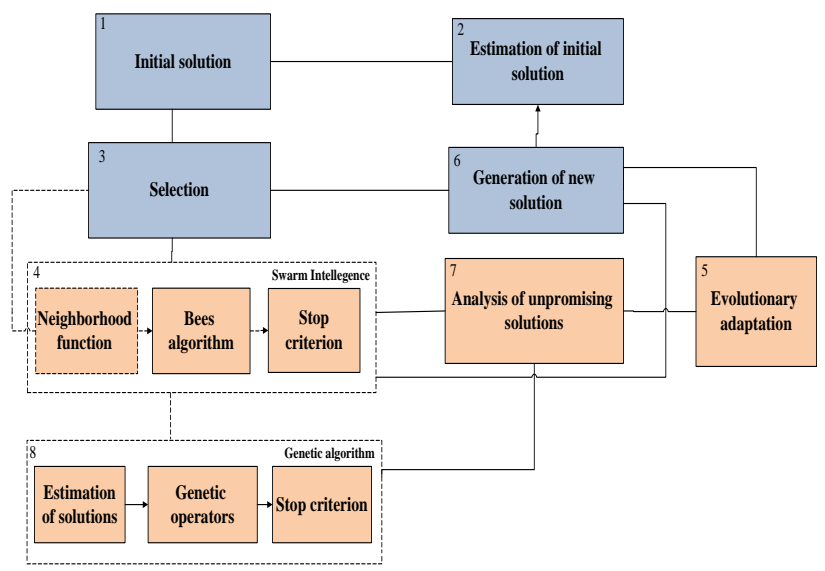

Fig. 1. Modified architecture of the hybrid approach

Let us describe the search process in detail.

At the first step the initial solution (Module 1) is generated and on the basis of the target function the best alternative solutions are selected (Module 3). In the Swarm Intelligence Module (Module 4) besides execution of the bees algorithm there are defined the neighborhood function and stop criterion. A number of iterations is denoted in initial data. When predetermined number of iteration is reached the best solutions are saved. Then, the best solutions are searched in new neighborhood. The selection criterion is a minimum value of the target function. After search of the best solutions, it is generated a solution matrix and the algorithm returns to the new iteration. The search neighborhood is formed on the basis of random transpositions in the chromosome. The transposition is a change of a couple of neighboring genes in the string. To find the value of the neighborhood function we need to estimate the quality of position and obtained solutions.

The next, in the Genetic Algorithm Module (Module 8) there are implemented the estimation of quality solution, realization of genetic operators and checking the stop criterion. Besides crossover operator, we suggest to use mutation and inversion operators. Consequently, we generate a new set of alternative solutions. The target function is calculated for each solution. Module of Evolutionary Adaptation (Module 5) is used to apply different adaptation mechanisms which changing the order of usage of different genetic operators. The Module 5 allows to change the current population of alternative solutions and create a new population.

The Module of Analysis of Unpromising Solutions (Module 7) collects solutions obtaining during the genetic algorithm execution. As a result of conducted analysis each chromosome has a definite rank (promising, unpromising, trivial etc.).

\section{MODIFIED STRUCTURE OF HYBRID SEARCH}

Consider a modified structure of hybrid search (Fig. 2).

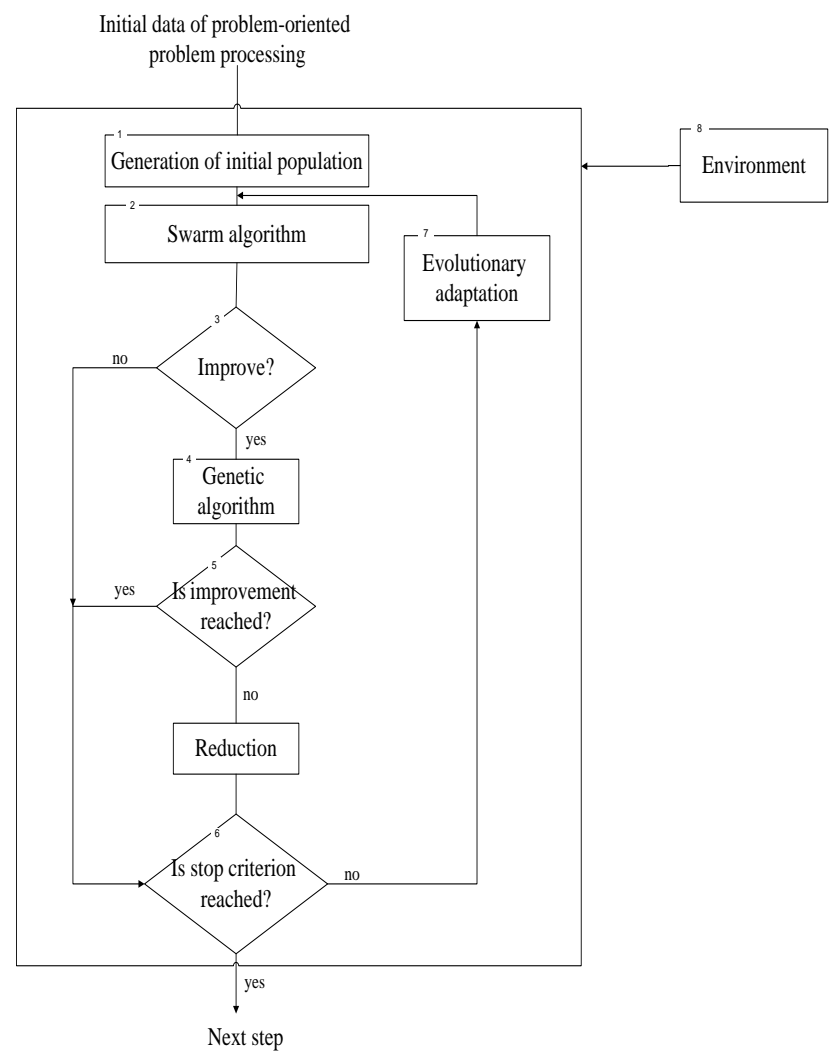

Fig. 2. The modified structure of hybrid search

Here we introduce adaptive probability to delete solutions with low value of the target function. The suggested structure contains main components of bioinspired search; to improve quality of search it is possible to add new algorithms, a module of evolutionary adaptation, a module of environment etc.

The modified structure of hybrid search involves the basic genetic algorithm. This structure allows to generate a population of alternative solutions and improve the quality of solutions at each iteration.

Here, an initial data involves parameters of circuit such as quantity of connections, quantity of elements and connectivity matrix. 
Next the bees algorithm is executed. This algorithm inspired by the behavior of bees in nature which is simulated in the following way. A free agent (bee) is in the hive until a forager give a signal by the so-called "wiggle dance". If this signal has been received then the agent moves toward to the source of nectar; otherwise - the agent became the forager after a while. . If the supposed source has nectar, the agent brings to the hive and simulates a "wiggle dance".

This strategy allows to find locally optimal solutions in appropriate time. After the bees algorithm execution, obtained results are analyzed in the "Improve?" Block. If improvement is necessary then the "Genetic Algorithm" Block is performed; otherwise - the "Stop criterion".

In the "Genetic Algorithm" Block there are applied selection methods and its modifications which, in the majority of cases, give an opportunity to solve the problem of preliminary convergence. In order to ensure good initial solutions we suggest to use genetic algorithms and a preprocessing heuristic.

Here, a stop criterion is a maximum number of iterations or convergence of the algorithm. Convergence of the algorithm is defined by comparison of target function values in several iterations. . The algorithm terminates in case of stabilization of this parameter.

It should be noted that evolutionary adaptation is applied only when the stop criterion is not reached and search is continued iteratively. Evolutionary adaptation is based on the generation of a new population.

So, major differences between the hybrid algorithm and others optimization methods is as follows:

- the hybrid algorithm provides a parallelizing of search process;

- genetic and evolutionary algorithms handle all admit solutions to obtain alternative ones;

- genetic and evolutionary algorithms are based on probabilistic, deterministic and hybrid mechanisms;

- the hybrid algorithm is a search strategy based on the calculation of the target function and do not require knowledge about additional information;

- the hybrid algorithm searches a local solution in the same way for different target functions.

As a result, to increase the efficiency of problem-oriented knowledge processing, it is reasonable to use evolutionary modeling, genetic search and adaptation and self-organization principles.

\section{EXPERIMENTS}

An algorithm, as a development object on one side and a tool for problem solution on the other, is also a subject of research. From the applied viewpoint the developer considers following questions:

- quality of solutions;

- execution time of the algorithm.
An algorithm is accurate if it finds $100 \%$ optimal solutions for any input data. The accurate solution can be obtained only by exhaustive search but this method is not suitable to high dimensionality NP-complete problems.

Computational complexity is defined by execution time of the algorithm on the determined set of input parameters. Consequently, computational complexity is a sum of product between one type operations and time needed for one operation of this time.

Time complexity is dependence of execution time of the algorithm on dimensionality of the problem. Time complexity of the algorithm is composed of time complexities of search methods included in the algorithm.

Let us denote a theoretical estimation of time complexity of the hybrid placement method. The initial solution can be obtained by a sequential algorithm with linear time complexity $\mathrm{O}(\alpha \mathrm{N})$ or by a blind search with time complexity $\mathrm{O}(\beta \mathrm{IN})$, where $\mathrm{I}$ is a number of iteration. As a result we noted that time complexity of the developed modified algorithm do not exceed the polynomial dependence and represented as $\mathrm{O}(\alpha \mathrm{N} 2)$ $\mathrm{O}(\beta \mathrm{N} 3)$, where $\mathrm{N}$ is a number of circuit elements.

Also we analyzed the dependence of execution time on number of iteration. Experiments were carried out on different test examples; size of population was equal to 50 ; probability of crossover operator $-85 \%$; probability of mutation operator 20\%; a number of GA colonies - 4. Average results are presented on Fig. 3.

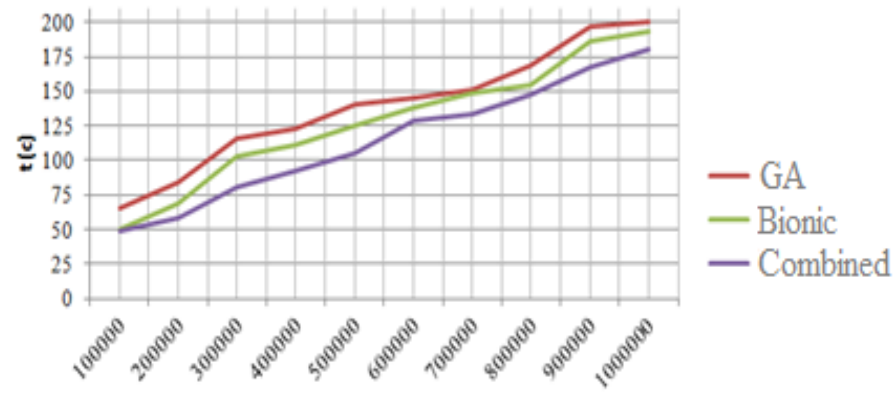

The number of circuit elements

Fig. 3. The dependence of execution time of the algorithm on size of circuit

We use a several test examples to evaluate efficiency of the developed method. . Efficiency of the algorithm refers to quality of solutions obtained with the use of this algorithm (Fig. 4).

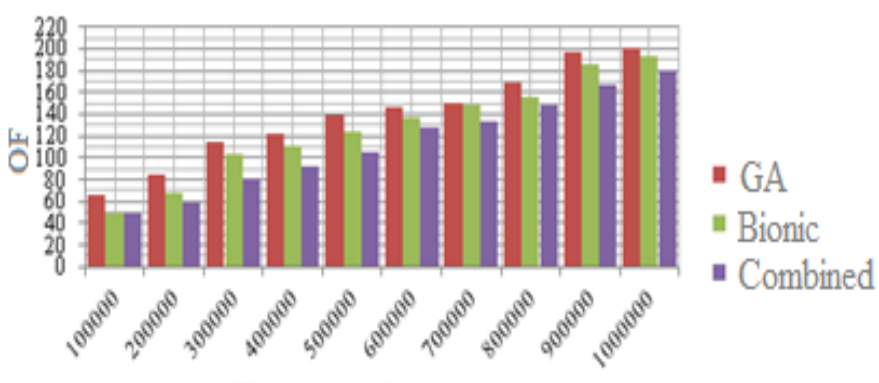

Fig. 4. Comparison of solutions quality 
On the basis of carried out experiments we can conclude that the developed hybrid search is more effective then GA and evolutionary algorithm for $15 \%$ and $10 \%$ correspondingly.

Obtained data is shown that genetic and evolutionary algorithm are more suitable for low dimension problems (up to 2000 elements), but for the high dimensional problems - the hybrid algorithm.

\section{CONCLUSION}

This work deals with hybrid approach, adaptation and selforganization for processing of problem-oriented knowledge. The main idea contains a sequential implementation of genetic and evolutionary algorithms. Also the example of algorithm was presented which investigated a search neighborhood by hybrid method. The formulation of the task of problemoriented knowledge processing during optimal solutions search was made. To increase the effectiveness of problem solving we used evolutionary simulation, genetic search, adaptation and self-organization. The modified structure of hybrid search was developed. This structure involves main components of bionic search and also there is an opportunity to add new algorithm, evolution adaptation and environment. Experiments were carried out which confirm that time complexity do not exceed polynomial dependence. The time complexity of the developed algorithm is represented as $\mathrm{O}\left(\mathrm{n}^{2}\right)$.

\section{ACKNOWLEDGEMENTS}

The study was performed by the grant from the Russian Science Foundation (project \# 14-11-00242) in the Southern Federal University.

\section{REFERENCES}

[1] J.P.Cohoon, J.Karro, J.Lienig, "Evolutionary Algorithms for the Physical Design of VLSI Circuits. Advances in Evolutionary Computing: Theory and Applications", Ghosh, A., Tsutsui, S. (eds.) Springer Verlag, London, pp. 683-712 (2003)
[2] G.F.Luger, "Artificial Intelligence. Structures and Strategies for Complex Problem Solving", 6th Ed. Addison Wesley, Boston MA (2009)

[3] V.V.Kureichik, V.M.Kureichik, S.P.Malioukov, A.S.Malioukov, "Algorithms for Applied CAD Problems. Berlin Heidelberg: SpringerVerlag”, pp. 487 (2009).

[4] C. J.Alpert, P.M. Dinesh, S.S.Sachin, "Handbook of Algorithms for Physical design Automation", Auerbach Publications Taylor \& Francis Group, USA (2009)

[5] V.V.Kureichik, D.V. Zaruba, "The bioinspired algorithm of electronic computing equipment schemes elements placement". In: 4th Computer Science On-line Conference, CSOC 20156 Silhavy. R., Senkerik. R., Oplatkova. Z. K., Prokopova. Z., Silhavy, P. (Eds.), vol. 347, pp. 51-58, Springer Verlag (2015)

[6] D.Y. Zaporozhets, D. Zaruba, V. Kureichik, "Hybrid bionic algorithms for solving problems of parametric optimization", J. World Applied Sciences, vol. 23, pp. 1032-1036 (2013)

[7] D.Y. Zaporozhets, D.V. Zaruba, V.V. Kureichik, "Representation of solutions in genetic VLSI placement algorithms", In: Proceedings of IEEE East-West Design \& Test Symposium - (EWDTS'2014) Kiev, Ukraine, September 26-29, (2014)

[8] E.V. Kuliev, A.N. Dukkardt, V.V. Kureichik, A.A. Legebokov, "Neighborhood Research Approach in Swarm Intelligence for Solving the Optimization Problems", In: Proceedings of IEEE East-West Design \& Test Symposium - (EWDTS'2014) Kiev, Ukraine, September $26-29$, pp. $112-115$ (2014)

[9] D. Zaporozhets, D. Zaruba, V.Kureichik, "Hierarchical approach for VLSI components placement", In: 4th Computer Science On-line Conference, CSOC 20156 Silhavy. R., Senkerik. R., Oplatkova. Z.K., Prokopova. Z., Silhavy. P. (Eds.), vol. 347, pp. 79-87, Springer Verlag (2015)

[10] S.K. Lim, "Practical Problems in VLSI Physical Design Automation", Springer Science + Business Media B.V, Germany, (2008).

[11] V.V. Bova, A.A. Lezhebokov, L.A. Gladkov, "Problem-oriented algorithms of solutions search based on the methods of swarm intelligence", J.World Applied Sciences, vol. 27, pp. 1201-1205 (2013)

[12] J. Kacprzyk, V.M. Kureichik, S.P. Malioukov, V.V. Kureichik, A.S. Malioukov, "Experimental investigation of algorithms developed. Studies in Computational Intelligence", 212, pp. 211-223+227-236 (2009) 